\title{
LA ASIMETRÍA CEREBRAL \\ Pautas y Ritmo en los procesos creativos
}

\author{
Aurelio del Portillo García \\ Profesor de Realización Audiovisual. Universidad Rey Juan Carlos.
}

\begin{abstract}
Resumen
Nuestra mente funciona estableciendo relaciones, contactos, hipervínculos, y mantiene un peculiar equilibrio entre los elementos que relaciona, entre las partes y el todo, entre la fragmentación y la unidad. En la interacción entre los hemisferios derecho e izquierdo del cerebro se produce la inevitable interdependencia entre las notas musicales y la música: las partes carecen de sentido sin la totalidad y viceversa. Este modelo puede ser aplicado a cualquier juego de percepciones, imaginaciones o concepciones que se construyen en la mente, en lo que se podría considerar un movimiento entre diferentes niveles o planos de realidad, como formas a partir de una más o menos intrincada arquitectura relacional de ideas, con su correspondiente correlato de significados intelectuales y emocionales. Se considera que la coherencia del resultado de cualquier proceso creativo se apoya precisamente en las pautas de relación que siguen las asociaciones de ideas y en su equilibrio y adecuación según un marco de proporciones al que se llama Ritmo, la razón de unidad en la que se relacionan y armonizan los diferentes fragmentos, elementos o signos con los que construimos textos, mundos, símbolos, imágenes, representaciones,...
\end{abstract}

\section{Palabras clave}

Pensamiento no verbal - Asimetría cerebral - Imaginación - Símbolos - Asociación de ideas Pautas - Ritmo 


\section{Abstract}

Our mind works building relations, connections, links... It maintains a peculiar balance between the elements that it relates, between the parts and the whole, between the fragmentation and the oneness. The necessary interdependence between musical notes and music takes place in the interaction between the right and left hemispheres of the brain: the parts have no sense without the totality and vice versa. This model can be applied to any relation of perceptions, imaginations or concepts, all of them under mental construction as forms, a relational architecture between ideas with their correspondence in emotional and intellectual meanings. It is considered that any creative process is supported by this special kind of balance and adjustment working according to the proportions that have been called Rhythm, the reason of unity in which the different fragments are related and harmonized.

\section{Key words}

Nonverbal thought - Cerebral asymmetry - Imagination - Symbols - Association of ideas - Rhythm

\section{Niveles de realidad}

“Temo que la palabra 'realidad' no constituye una característica ordinariamente definible de las cosas a las que se la aplicamos, sino que se la usa como si fuese una especie de halo celestial. Dudo mucho que alguno de nosotros tenga la menor idea de lo que significa la realidad o la existencia de algo que no sea nuestro propio ego” (EDDINGTON, 1931; pg. 419).

Nadie es consciente de que sueña cuando está dormido, salvo en el poco habitual estado de sueños lúcidos (VARELA, 1998; pg. 107). Quizás por eso muchos, por no decir casi todos, reaccionan con diferentes grados de rechazo ante la idea de que lo que consideramos nuestro estado cotidiano de vigilia no es lo "real”, sino un estado más de la mente que puede ser trascendido también por un despertar. Sin embargo esto ha sido afirmado con toda rotundidad por algunos de los grandes sabios 
del siglo XX, como Krishnamurti, Nisargadatta, Ramana Maharshi o, en nuestro entorno más cercano, Alan Watts, Ken Wilber, Eckhart Tolle, Consuelo Martín,...

“El tiempo es la clave del conocimiento distorsionado de esta experiencia múltiple a la que llamamos la realidad. Y mientras permanezcamos en esta conciencia temporal, la verdadera realidad estará vedada para nosotros porque es incognoscible para el conocimiento en el tiempo. La tensión de conciencia hacia el objeto rompe la unidad original y entonces es cuando vemos el espectáculo del estado de vigilia ordinario, donde lo real aparece fragmentado en mil pedazos y separado en momentos” (MARTÍN, 1998; pg. 91).

Pero el hecho de que lo que llamamos realidad no es más que una forma de considerar comportamientos de energía también ha sido de alguna forma demostrado por la física, por la tan sacralizada ciencia occidental, al confirmar que la materia, el tiempo y el espacio no tienen entidad propia como objetos independientes, sino que existen tan sólo en un complejo sistema de relaciones en las que no se encuentra ninguna partícula material a partir de la cual se hubieran podido “edificar”. Por eso este texto no va a diferenciar las diferentes construcciones mentales como reales o ficticias, sino que va a considerar todo el proceso de la percepción sensorial, la percepción mental, la imaginación, las ideas, los lenguajes, las emociones, etcétera, como integrantes de un mismo sistema en el que se pueden contemplar diferentes niveles o planos de realidad, sin intentar por el momento ir más allá en búsqueda de lo que pudiera ser calificado sin ningún tipo de dudas como lo real.

Ya se ha considerado anteriormente el fenómeno de la cinematografía como un "soñar despiertos" (PORTILLO, 2000). El acercamiento que se hace en esta ocasión a los procesos creativos está en ese marco de observación de los mecanismos de la mente humana por los que se relacionan los elementos de construcción de un texto con sus significados intelectuales y emocionales, tanto si se trata de lenguajes construidos con palabras como con otro tipo de signos o formas significantes. Pongamos un ejemplo: cuando se realiza un encuadre, se selecciona con él una forma visual, y posteriormente son tratados los contrastes o tonos dominantes de esa imagen dotándola de una expresión estética que se canaliza para su lectura en un soporte determinado, se crea una representación, una interpretación en la que se conjugan los diferentes mecanismos del cerebro capaces de articular los movimientos o transformaciones de la luz en formas que significan algo. No importa ahora de qué “algo” se está hablando, sin duda inseparablemente vinculado a los más diversos condicionantes culturales, convencionales, contextuales, etcétera. Pero sí es importante 
señalar que no se habla tan sólo de significados cerrados, sino más bien de un amplio campo de posibilidades que van mucho más allá de la apariencia física de las cosas y de sus representaciones. Antoine de Saint-Exupéry escribió en El Principito que "no se ve bien sino con el corazón, lo esencial es invisible a los ojos”. Quizás una de las tareas fundamentales de cualquier trabajo creativo consiste en destacar aspectos esenciales buscados en el alma de las cosas. La mirada, pues, en este sentido, no puede ser meramente física. ¿En qué otro plano de las realidades de la mente puede estar arraigada? ¿Son los sentimientos, por ejemplo, también mentales? ¿Acaso podemos considerar por separado lo emocional? Parece evidente que toda emoción está relacionada con una construcción mental, como también lo es que existe lo que ha sido denominado como "inteligencia emocional” (GOLEMAN, 1996). Se puede inferir de todo ello que hay diferentes comportamientos de la mente humana en sus formas de relacionar elementos de todo tipo aparentemente fragmentados o separados y que se puede buscar en esos diferentes niveles de realidad una integración de formas más o menos complejas y sutiles. Desde las líneas con las que se representa la apariencia del espacio hasta las vivencias de un personaje con las que se representa un arquetipo social, pasando por los colores con los que se puede asociar un estado anímico. Y así un largo, ¿infinito?, etcétera.

Decía Pierre Schaeffer en su Tratado de los objetos musicales que "el secreto está entonces en mantener al oído constantemente activo sin violentarlo”, refiriéndose a la necesidad de despertar y mantener desde una composición musical la atención de quien la escucha, en relación con las duraciones musicales (SCHAEFFER, 1988; pg. 155). Pero ¿qué queremos decir con atención? ¿Qué actividad mental supone esa proyección hacia algo susceptible de ser comprendido, sentido, interpretado? A simple vista parece ya que no se trata sencillamente de yuxtaponer signos para asociar significados concretos, sino de integrar lecturas mucho más complejas y diversas en las que intervienen niveles mentales capaces de asociar en amplias formas todos los elementos que intervienen en la construcción, interpretación y comprensión de un texto. Todo ello se amplía y despliega en territorios que superan lo estrictamente racional, aunque se plantea por el momento en el ámbito de las diversas actividades y funciones del cerebro.

\section{La asimetría cerebral}

“Ahora sabemos que a pesar de nuestra normal sensación de ser una sola persona, un solo ser, nuestro cerebro es doble, y cada mitad tiene su propia manera de conocer, su propia manera de percibir la realidad” (EDWARDS, 1989; pg. 47). 
A diferencia del de los animales, el cerebro humano no es simétrico. Sus dos hemisferios cumplen funciones distintas y en la complicadísima red de conexiones que se establece entre ambos surge la integración de formas complejas que van más allá de la interpretación de signos aislados. Esa asimetría cerebral comienza en los Australopitécidos, según se demostró en 1968, y continúa evolucionando hasta el cerebro humano. Y aún continúa porque, según parece, esa evolución no ha finalizado (ROJO-SIERRA, 1984; pgs. 120 y sig.). Además de estar relacionada con la dextralidad (uso dominante de la mano derecha), con el cruce de los hemicampos visuales de ambos ojos, y otros comportamientos y actividades del ser humano, la asimetría cerebral supone una diferencia de modos de interpretación de las percepciones y del procesamiento de datos que nos resulta de enorme interés para comprender el funcionamiento de la mente en general y, de forma más concreta, el mecanismo mental en los procesos creativos:

"Las personas creativas han reconocido las diferencias entre el proceso de reunir información y el de transformarla creativamente. Los últimos descubrimientos sobre el funcionamiento del cerebro comienzan a arrojar luz sobre este proceso dual. Conocer ambos lados del cerebro es un paso importante para liberar nuestro potencial creativo" (EDWARDS, 1994; pg. 42).

A partir de la observación de ciertas patologías se ha ido precisando qué funciones son propias de la actividad de cada hemisferio. Sabemos que la función del lenguaje, por ejemplo, pertenece fundamentalmente al hemisferio tipo izquierdo (algunas personas tienen las funciones de los hemisferios cerebrales invertidas, como es el caso de los zurdos). Hasta tal punto ha sido valorada esa actividad dominante en algo de tan enorme importancia para el ser humano, que el hemisferio tipo derecho fue sido considerado hasta hace muy poco como subordinado al izquierdo (EDWARDS, 1994; pg. 43). Sin embargo, la constatación de la función integradora o globalizadora del hemisferio derecho ha ido modificando esta valoración:

"Los datos indican que el hemisferio no verbal subordinado se especializa en la percepción del conjunto y que su función consiste principalmente en sintetizar la información que llega. El hemisferio verbal dominante, por el contrario, parece actuar de un modo más lógico, analítico, al estilo de un ordenador. Su lenguaje no es apto para las rápidas síntesis que realiza el hemisferio subordinado” (LEVY, 1968; pg. 1151). 
Digamos que el hemisferio izquierdo lee las notas musicales, pero es el hemisferio derecho el que "ve” la música. Es el hemisferio derecho el que integra formas, interactuando con el hemisferio izquierdo que lee los elementos con los que esas formas se construyen. Esto supone también que “el hemisferio izquierdo analiza en el tiempo, mientras que el hemisferio derecho sintetiza en el espacio” (LEVY, 1974; pg. 61). La secuencialidad y la simultaneidad no son percibidas por el mismo tipo de actividad cerebral. Y esta desigualdad resulta muy interesante.

¿A qué actividad cerebral corresponde entonces la contemplación estética o emocional de una imagen o de una idea? ¿Puede analizarse de la misma forma la visión parcial o fragmentada de las cosas frente a la visión integradora o totalizadora? ¿Es el hemisferio derecho, hasta hace poco, como ya se ha dicho, valorado como subordinado al izquierdo, más espiritual? Interesantes preguntas. Aunque se puede plantear ya de entrada una observación problemática, y es que quizás no todo lo que concierne a una experiencia estética sea asunto sólo de las mecánicas cerebrales, de sus comportamientos físicos, sino que pudiera haber algo más allá de la materialidad cerebral. De hecho parece lógico pensar que todo lo que parta de los mecanismos cerebrales que tengan relación con lo ya aprendido, con la memoria, pueda estar altamente condicionado y limitado como para ser capaz de actividades realmente creativas, de crear algo nuevo. Esto nos aproxima a un ya viejo conflicto planteado entre los conceptos conciencia, mente y cerebro. No pocos son muy críticos con la posibilidad de admitir que haya mente más allá del cerebro (BUNGE, 1988). El debate es posiblemente de los que más han sido cuestionados en el campo de la psicología y la filosofía y ha sido objeto de muchos estudios (ARCAS, P. y otros). También se han cuestionado las limitaciones del pensamiento para la comprensión de realidades trascendentes (BOHM y KRISHNAMURTI, 2001); (MARTÍN, 1999). Parece claro que, de la misma forma que se han planteado diferentes niveles de realidad, también pueden plantearse diferentes niveles de conciencia:

“1: Nivel sensorio-físico, 2: Nivel fantásmico-emocional, 3: Mente representativa, 4: Mente regla-rol, 5: Nivel reflexivo-formal, 6: Nivel visión-lógico, 7: Nivel psíquico, 8: Nivel sutil, 9: Nivel causal, 10: Nivel último”(WILBER, 1994; pg. 20).

No parece sencillo definir de forma racional y científica los límites y características funcionales de la conciencia, quizás porque, sencillamente, escapa al ámbito de lo razonable y no ha podido ser definida de ninguna manera como una entidad material, sin haberse llegado más allá de comprobar en qué variables cuantitativas y cualitativas se producen los fenómenos psíquicos que se asocian con ella (PÖPPEL, 1993). Pero de forma intuitiva sí puede valorarse el alcance que tiene la diferente visión e integración de estímulos y percepciones en la conciencia para acercarnos a los procesos en 
los que esos estímulos, datos, signos o informaciones son articulados y modificados con una finalidad creativa. Es decir, cuando una mente humana introduce en el procesamiento de formas visuales y/o sonoras, incluidos los elementos básicos de cualquier lenguaje que con ellas se construyen, algo más que una secuencia imitativa: una interpretación en la que se involucra directamente la personalidad creadora, la mente creativa. En este tipo de procesos las realidades materiales de alguna manera son trascendidas, cambian de nivel (BLAY, 1992). De la misma forma que unas vibraciones sonoras pueden ser definidas y nombradas como notas musicales, pero eso no las convierte en música. Las casi infinitas posibilidades de ser relacionados esos elementos básicos, físicos, puede elevar su entidad a algo superior: la forma musical. Y más allá de ella a la posible emoción estética que proviene de la inspiración que dio lugar a ese trabajo. Se vislumbra en todo ello una actividad mental basada en las funciones del hemisferio derecho, en la integración de formas, mucho más allá de la secuencialidad lógica de los signos. Algo de totalizador hay en todo proceso creativo, al menos en esa diferente forma de percibir la realidad a la que hacía referencia la cita de Betty Edwards con la que se encabeza esta sección.

No sería justo despreciar el valor del pensamiento como mecanismo cerebral de tanto alcance para la actividad humana, pero todo el que haya probado a tocar en el piano una obra moderadamente compleja de contrapunto de J. S. Bach sabe que no se puede pensar en ello mientras se ejecuta. Primero se ha leído el lenguaje de esa composición, luego se ha ejercitado el movimiento corporal que posibilita su ejecución, pero en el momento de ser comprendida y en el de convertirse en música viva la preponderancia dominante del hemisferio izquierdo parece casi desaparecer. Sin duda en la mente del compositor, como en la de un escritor o un director de cine, también se da este fenómeno en el que una ordenación de elementos aislados está guiada por una visión global sin la cual el pensamiento analítico quedaría absolutamente huérfano. Otra actividad mental-cerebral actúa, en la que, por cierto, parece también desvanecerse, atenuarse, o incluso desaparecer ese mecanismo de autoconciencia racional al que se ha llamado "ego”. Cuando el pensamiento se detiene y la mente se vuelve silenciosa, aunque muy lúcida y activa, algo más que el pensamiento desaparece.

Probablemente, cuando decimos en danza, música, y quizás en todas las expresiones artísticas, que hay que "dejarse llevar” nos estamos refiriendo a este cambio de las posiciones mentales. La inspiración, el abrirse a algo nuevo, parece pasar también por la debilitación previa del dominio de lo conocido, de los mecanismos dominantes del pensamiento, de esos ancestrales intentos de la humanidad por atrapar en algún tipo de red mental toda realidad cognoscible. 


\section{Redes y trampas}

“Cuando intentas agarrar algo que ondula, es difícil, ¿no es cierto? Intentas agarrar un pez y el pez ondula, y se te escurre. ¿Y qué haces para cazarlo? Utilizas una red. ][La red es algo regular, puedo numerar sus agujeros, tantos agujeros verticales, tantos agujeros horizontales. Si puedo numerarlos, puedo señalar exactamente la posición de cada onda, en términos de cada uno de los agujeros de la red. Y he aquí el comienzo del cálculo, el arte de medir el mundo” (WATTS, 1995; pg. 27).

Sabemos de los intentos de la humanidad por someter al dominio de los números incluso algo tan difícil, ¿imposible?, de definir como es la belleza. Ahí tenemos el ejemplo claro del número $\Phi$, el número de oro (GHYKA, 1968). Quizás en esa misma línea esté el permanente intento humano de nombrar (desde siempre me ha fascinado que número en francés se diga "nombre”), de atraparlo todo en palabras, esos signos pensados para la imposible tarea de hacer material toda idea y poderla de alguna forma poseer:

“¿Cómo puedes tú

tener un nombre entre los nombres?

Ni aunque sea un verbo

que diga "Pasó",

"Pasará”, "Pasara",

¿cómo pueden hablar de ti los hombres?

Es mentira en flor;

semilla es de sus errores:

porque, si te nombran,

parece que ya

saben lo que eres,

y hasta pueden creer que te conocen; (...)”

(GARCÍA CALVO, 1979; pg. 13)

Cabe hacerse la pregunta de por qué se pretende tal cosa. Y, en todo caso, asumiendo que se quiere nombrar y numerar toda realidad, incluso si su materialidad fuera de otra forma imposible, esas realidades que se quieren atrapar han sido traídas de alguna manera a la conciencia, han sido concienciadas o, al menos, intuidas. ¿¿De qué mecanismo mental estamos entonces hablando? 
¿Cuáles son sus objetos? ¿Puedo contemplar algo sin pensar en ello, antes de nombrarlo? Incluso se plantea el tiempo, en su dimensión psicológica, como una forma de explicar, de desplegar realidades, y se ha definido su entidad como una creación del movimiento mental del pensar, como algo fabricado por el pensamiento y de alguna manera connatural con él (KRISHNAMURTI y BOHM, 1996). No se va a entrar en esta ocasión en el fondo de la idea, que seguramente pasa por valorar de qué antiguos miedos proviene esa pulsión imperiosa del ser humano por dominar todo tipo de realidades en las redes de múltiples lenguajes y representaciones mentales, pero sí se plantea como necesario precisar que no se está hablando de una sola modalidad del pensar, del pensamiento verbal, sino de otras muchas que pueden adquirir matices incluso más sutiles y por lo tanto más cercanos a las formas mentales implicadas en los procesos creativos. Estamos acostumbrados a tener en una muy alta estima el acto de pensar como algo definitorio de la categoría y dignidad humana, pero en muchos casos puede que no seamos conscientes de que al mismo tiempo estamos encerrándonos en nuestra propia trampa, al limitar nuestra experiencia al ámbito de estructuras conocidas y cerradas con las que de alguna manera nos hemos acostumbrado a filtrar e interpretar el mundo y la vida. Se habla, pues, para limitar la limitación, de diferentes tipos de pensamiento tales como el pensamiento visual (ARNHEIM, 1986), el pensamiento corporal o el pensamiento dimensional (ROOT-BERNSTEIN, 2002, pg. 197), aunque como denominador común estará siempre la figura limitada del pensador:

“El cambio supone cierto lugar en que me sitúo y desde donde veo desfilar las cosas; no hay acontecimientos sin un alguien al que ocurren y cuya perspectiva finita funda la individualidad de los mismos” (MERLEAU-PONTY, 1975; pg. 419).

Desde la atalaya de la visión personal se puede tener una cierta perspectiva de las cosas, incluso de uno mismo. Pero cada vez es más visible la existencia de otra forma de enfrentarse a la realidad, a las realidades, en una dimensión que ha sido elocuentemente llamada 'transpersonal' (GROF, 1988). Parece que algo escapa de la trampa, de las redes del pensamiento-lenguaje-persona. Algunos valores incluso se sitúan más allá de los condicionantes, no ya sólo personales, sino también culturales o de contexto social, en los terrenos de la 'filosofía perenne’ (HUXLEY, 1977). Cuando una obra de arte se dice que llega al corazón, o, simplemente, que llega a las profundidades interiores de quien la contempla, ¿de qué sustancia se habla? ¿Se está diciendo quizás que pueden conectarse visiones transpersonales en un mayor o menor grado de abstracción? ¿Es ésa la dimensión divina del arte? ¿Por eso lo llamamos creación? ¿Es esto la inspiración? Indudablemente no se pretende en este texto dar respuesta a estas difíciles cuestiones. Simplemente se mira de forma reflexiva en la 
dirección hacia la que apuntan algunos razonamientos y vivencias. Está claro que sin nuestra percepción sensorial y las interpretaciones que a partir de ella elaboran nuestros sistemas de pensamiento racional, la vida del ser humano carecería del sentido que cobra como proceso de aprendizaje basado en las relaciones. Pero algo de esto que aquí se analiza ahora apunta más hacia un mundo interior no supeditado permanentemente a los estímulos exteriores. Y parece especialmente importante esta reflexión para observar con mucha atención y cuidado esa posición mental en la que se gesta un proceso creativo ante la hoja en blanco, la pantalla en blanco, la propia mente en blanco, y desde allí iniciar, gestar, alumbrar, nacer. ¿Quién mira qué para que eso suceda?

\section{La forma de la nada}

Este subtítulo tiene una intención metafórica. No se habla de una nada absoluta, pero sí de una cierta nada o vacío desde donde surge algo nuevo, una visión o idea. Decía Platón que “conocer es recordar" refiriéndose al ámbito de las ideas inmanentes que nuestra mente redescubre, comprende o trae al campo de lo consciente en su devenir vital. La mente da forma a una idea. Puede que lo haga basándose en algo conocido de una manera más o menos consciente, pero también puede que parta de un nivel tal de inconsciencia que aquello en lo que arraiga su proceso de creación o recreación no pueda ser considerado como una simple activación de la memoria. Incluso aunque esté relacionado con ella puede presentarse como un descubrimiento realmente innovador. Resulta muy interesante en este sentido una anécdota de Giacometti:

“Comenzó a pintar una vez más, pero después de unos pocos minutos se volvió hacia donde había estado el busto, como si fuera a examinarlo de nuevo, y exclamó: ‘¡Oh, ha desaparecido! Pensé que estaría allí todavía, pero ha desparecido’. Aunque le recordé que Diego se lo había llevado, dijo: 'Sí, pero pensé que estaba allí todavía. Miré y de pronto vi el vacío. Vi el vacío. Es la primera vez en mi vida que tal cosa me sucede’ " (ARNHEIM, 1986; PG. 101).

¿Cuáles son los límites de esta experiencia? ¿Es similar a la ausencia de un ser querido que se percibe como absoluta abstracción sin forma física? ¿Intuimos los vacíos que conforman las limitaciones de la existencia y buscamos algo que los complete para culminar cierta plenitud? No parece plausible que este tipo de visión se limite a las formas físicas, sino que parece razonable ampliarlo a todos los niveles de la mente y de la conciencia, a todos los niveles de realidad a los que 
ya se ha hecho referencia. En un sentido estrictamente material, asumiendo la relatividad del concepto de materia al que antes se ha hecho alusión, los comportamientos de ciertas vibraciones o ritmos de las diferentes energías que constituyen el mundo fenoménico entren en relación con otro tipo de vibraciones y ritmos energéticos con los que funcionan los mecanismos cerebrales. En esa arquitectura de relaciones se configura la percepción humana, pero también de forma similar otros procesos mentales como la imaginación, la fantasía, la ensoñación, todos ellos basados, según parece, en cierta actividad de movimientos de energía que interconectan las diferentes zonas y funciones del cerebro (SAKOTA, T. y TAKAO, M., 1994).

La cuestión es valorar hasta qué punto es necesaria la reiteración de patrones a la que se llama materia para que las energías que se relacionan en un proceso creativo tengan lugar a nivel mental, teniendo en cuenta que se está hablando de actividad cerebral y por lo tanto también de ciertos patrones de comportamiento de la energía que pueden ser igualmente considerados como materiales. Pero ¿cuál es el origen del proceso? ¿Qué lo inspira? ¿De qué se parte? Se vislumbra en el intento de respuesta a estas interrogaciones esa 'nada', esa pantalla en blanco sobre la que se visualiza o concibe una forma que agrupa elementos y factores muy diversos en no menos diversos niveles, desde el agrupamiento de las vibraciones mecánicas que constituyen el sonido hasta los difícilmente razonables ámbitos de la inspiración, pasando por el vasto campo de posibilidades que se abre en la interrelación entre ideas, emociones y experiencias estéticas. Un texto pretende, y a veces lo consigue, hacer perceptible, palpable, ese universo multidimensional. En él se despliega, desde un punto de vista semiológico, la red de significados que nos permite interpretarlo. Todos estos procesos están regulados por algo más que la configuración de nuestro cerebro y del desarrollo de los diferentes lenguajes que de ella se derivan. También interviene el contexto social, la cultura, y los múltiples condicionamientos que incluso de alguna manera predeterminan muchas facetas del ser humano.

“El grado de procesamiento sensorial o ‘interpretación’ no sólo depende de la complejidad del mensaje sensorial, sino también de la estructura del cerebro, no sólo de la que por nacimiento poseemos, también de la organización adquirida a lo largo del desarrollo del animal en su medio” (BUNGE, 1988; pg. 114).

Pero una vez considerada la posibilidad de lo transpersonal, y de otros conceptos cercanos como puede ser el de inconsciente colectivo (RETAMALES, R., 2004), podría establecerse un paralelo de similitudes entre los procesos mentales personales y los que podrían darse más allá de las fronteras de la persona. Al menos desde el punto de vista de que se trata de una compleja trama de relaciones, 
agrupamientos y asociaciones de ideas, símbolos, abstracciones, emociones, percepciones, interpretaciones, imágenes, textos,... Y entre todas esas relaciones se crean determinadas pautas.

\section{Pautas y ritmo}

“El proceso de formación de pautas no es más que el fruto de combinar dos o más elementos estructurales y/u operaciones funcionales. La yuxtaposición coherente de dos elementos u operaciones da origen a una pauta sintética que puede ser mucho más -y muy diferente, por cierto- que la simple suma de sus elementos constitutivos” (ROOT-BERNSTEIN, 2002; pg. 146).

Se conoce con claridad el planteamiento de la psicología de la forma que define esa entidad superior a la suma de los elementos que la constituyen. En niveles muy básicos es fácilmente comprensible que un cuadrado tiene un valor propio más allá de la suma de cuatro líneas rectas, al igual que una forma musical como tal es lo que es y no simplemente una coincidencia en el tiempo de vibraciones independientes. Esa coherencia en la relación a la que se refiere la cita que precede a estas palabras responde a un amplísimo campo de posibilidades. Una vez agrupadas operaciones mentales de cualquier índole en una forma coherente ya no importa si estamos uniendo emociones y colores, sonidos y metáforas poéticas u objetos de conciencia sutiles con articulaciones lógicas.

“Los artistas, los músicos, los bailarines, los físicos, los matemáticos y los inventores conciben y crean de continuo nuevas pautas con todo tipo de material -ya sea físico o mental- para acabar descubriendo, con mucha frecuencia, que previamente ya existían, pero habían sido soslayadas” (ROOT-BERNSTEIN, 2002; pg. 147).

Lo que ahora interesa es precisamente ese mecanismo de relación en el que los fragmentos aislados que pueden ser articulados por el hemisferio izquierdo del cerebro, o por la actividad correspondiente a esa función en otros niveles mentales, adquieren la entidad de una forma nueva, de alguna manera independiente, con vida propia, más allá de la suma de esos elementos constitutivos. La pauta que los relaciona se hace reconocible y protagonista de la posibilidad de que cualquier creación sea comprendida y transmitida la idea y/o emoción que lleve asociada. Se llama ritmo a esa coherencia que mantiene un vínculo de unidad entre los elementos o fragmentos de un conjunto o 
totalidad. Y esas globalidades, esas formas o pautas, ese ritmo, es el ámbito funcional del hemisferio tipo derecho, de la actividad cerebral, mental, que se suponía subordinada al proceso analítico secuencial del hemisferio tipo izquierdo. Relacionada sí, pero ¿subordinada? No debería estar el lenguaje por encima de lo que como vehículo transmite y canaliza. Una vez más se recuerda aquello de que “cuando el sabio señala la luna el necio se queda mirando el dedo”. Sería una limitación reduccionista imperdonable. ¡Cuántas veces se pierde la razón en sus propios vericuetos sin dar un paso atrás para ver el plano general del proceso y descubrir en su totalidad a qué apunta, a qué se refiere, de qué habla! Los procesos creativos no pueden ser considerados como un álgebra de posibilidades combinatorias entre los elementos formales con los que trabajan, sino que deben incluir en su mirada también el espíritu que los inspira, la forma integral que abarca todos los niveles implicados.

Ya se ha observado en este texto que incluso la propia materia no es más que un comportamiento de la energía que sigue determinadas pautas. La física, en su inmersión hacia las partículas mínimas, indivisibles, de lo material, ha llegado a definir esa prácticamente inexistente materialidad como una relación de probabilidades imposible de determinar, como una vibración. Buscando como átomo (no divisible) algo parecido a una piedra muy pequeñita con la que se hubiera construido el cosmos, se ha encontrado con una música. "La teoría cuántica nos fuerza a ver el universo no como una serie de objetos físicos, sino más bien como una complicada telaraña de relaciones entre las diversas partes de un todo unificado” (CAPRA, 1997; pg. 178). Si se sigue la pirámide de niveles de realidad y de conciencia a la que se ha hecho referencia se puede encontrar el ámbito holístico de estas relaciones. La música de las esferas puede ¿descender? a las partículas subatómicas y las asociaciones de ideas guardar armonías rítmicas con ese estado interior capaz de descubrir la belleza. En el camino hay muchas posibilidades, y en ellas se mueven muy probablemente la creación artística y la intelectual. Las redes conectivas de la mente esteblecen la pauta de relaciones en las que se apoya todo proceso intelectivo y también, por supuesto, el espacio inmenso de la creatividad. Resulta sencillo descubrir la pauta que relaciona acentos en la secuencia de un verso, y siguiendo por ese camino también la posibilidad poética de todo lenguaje. El ritmo como razón de unidad y equilibrio en las reiteraciones y proporciones es reconocible en todos los ámbitos de las diferentes realidades, y podrían valorarse innumerables ejemplos. Uno de ellos puede ser el de los relatos audiovisuales (PORTILLO, 2005). Y también, como ya se ha dicho, cualquier creación artística, intelectual o científica que se basa en el reconocimiento de las pautas en las que pueden conectarse lenguajes aparentemente diversos: "Cuando observamos un lenguaje corporal, lo que vemos es un sistema que mantiene algunos paralelismos con el lenguaje hablado” (KNAPP, 1982; pg. 203). Hay 
un orden implicado en todos los sistemas de relación que nuestras formas de organización mental patentizan:

"Sin nuestros principios de organización los objetos no podrían ser objetos, y, por consiguiente, los cambios fenoménicos producidos por tales cambios de la estimulación serían tan desordenados como los mismos cambios de la estimulación. De modo que aceptamos el orden como una característica real, pero no necesitamos un agente especial para producirlo, ya que el orden es consecuencia de la organización y la organización el resultado de fuerzas naturales” (KOFFKA, 1973; pg. 210).

Los fenómenos de la naturaleza, el funcionamiento de nuestro propio universo corporal, nuestra relación intelectiva con el espacio y el tiempo, todo ello parece responder a ciertas claves de orden. De nuevo la mente debe asumir el reto de soltar amarras para que no se impongan solamente los modelos repetitivos del pensamiento mecánico en su lectura-escritura del mundo, y, como en un viaje astral, situar su punto de vista en una perspectiva global que lo integre todo, incluso a sí misma, rompiendo las barreras entre el que mira y lo mirado quedando sólo la mirada. La creatividad no es sólo una actitud o posición mental que abre las posibilidades de una visión nueva sobre algo que ya existe o que genera algo totalmente nuevo, sea cual sea su nivel de realidad. Es también una forma de relacionar que sirve como pauta para la interpretación de los textos y lenguajes en los que se expresa: también la lectura es creativa. Parece que algún orden inspiró a Bach o a Van Gogh cuando abrían las ventanas de su mente hacia dimensiones poco exploradas, y eso mismo inspira a quien contempla sus obras. Visto de esta forma, y con la misma intención holística que se ha aplicado al conjunto de estas reflexiones, observamos los elementos que constituyen la realidad integral de una creación artística de tal manera que ninguno de ellos finaliza en su entidad aislada sino que cobra valor y sentido en su participación en el conjunto. También entre los diferentes factores y actores del proceso comunicativo.

El descubrimiento desde esta toma de conciencia de las infinitas posibilidades de creaciónrecreación-reconocimiento de relaciones entre todo lo que existe o puede llegar a existir es suficiente motor como para emprender el viaje. 


\section{Bibliografía}

- ARCAS, P. y otros: El problema mente-cerebro (seminario de filosofía), en Indexnet, Santillana.

- $\quad$ ARNHEIM, R., 1986: El pensamiento visual, Paidós, Barcelona.

- BLAY, A., 1992: La personalidad creadora, Índigo, Barcelona.

- $\quad$ BOHM, D. y KRISHNAMURTI, J., 2001: Los límites del pensamiento, Kairós, Barcelona.

- BUNGE, M., 1988: El problema mente-cerebro, un enfoque psicobiológico, Tecnos, Madrid.

- CAPRA, F., 1997: El tao de la física, Sirio, Málaga.

- DAVIS, F., 1976: La comunicación no verbal, Alianza, Madrid.

- DOSSEY, L. 1969: Tiempo, espacio y medicina, Kairós, Barcelona.

- EDDINGTON, A., 1931: The nature of Physical World, MacMillan, Nueva York.

- EDWARDS, B, 1994: Aprender a dibujar con el lado derecho del cerebro, Urano, Barcelona.

- GARCÍA CALVO, A., 1979: Libro de conjuros, Lucina, Zamora.

- GHYKA, M., 1968: El número de oro (2 vols.), Poseidón, Buenos Aires.

- GOLEMAN, D., 1996: La inteligencia emocional, Kairós, Barcelona.

- GROF, S., 1988: Psicología transpersonal, Kairós, Barcelona.

- HOLST, I., 1973: Conducting a choir, Oxford University Press, Oxford.

- HUXLEY, A., 1977: La filosofía perenne, Edhasa, Barcelona.

- KNAPP, M.L., 1982: La comunicación no verbal, Paidós, Barcelona.

- KOFFKA, K., 1973: Principios de psicología de la forma, Paidós, Buenos Aires.

- KRISHNAMURTI, J. y BOHM, D., 1996: Más allá del tiempo, Kairós, Barcelona.

- LEVY, J., 1968: “Differential Perceptual Capacities in Major and Minor Hemispheres” en Proceedings of the National Academy of Sciences, vol. 61, 1137-1160, USA.

, 1974: “Psychobiological Implications of Bilateral Asymmetry”, en DIMOND, S.J.

and BEAUMONT, J.G. (eds.): Hemisphere Function in Human Brain, John Wiley and Sons, Nueva York.

- MARTÍN, C., 1998: Conciencia y realidad, Trotta, Madrid. , 1999: El silencio creador, Mandala, Madrid.

- MERLEAU-PONTY, M., 1975: Fenomenología de la percepción, Península, Barcelona.

- PÖPPEL, E., 1993: Los límites de la conciencia: realidad y percepción humana, Círculo de lectores, Barcelona.

- PORTILLO, A., 2000: Soñar despiertos: las fronteras de lo real en Babab, revista de cultura en Internet, $n^{\circ} 4$. 
, 2005: "Estructura rítmica del relato audiovisual, desde el guión al montaje”, comunicación en el I Congreso Internacional de Análisis Fílmico, Madrid (libro de actas).

- RETAMALES, R., 2004: “Los arquetipos y lo inconsciente colectivo, comentario al tomo 9/I de las obras completas de C.G. Jung”, en Fundación Carl Gustav Jung, www.fcgjung.com.es

- ROJO-SIERRA, 1984: La asimetría cerebral y la experiencia psicológica y patológica del tiempo, Gregori, Valencia.

- $\quad$ ROOT-BERNSTEIN, R. y M., 2002: El secreto de la creatividad, Kairós, Barcelona.

- SAKOTA, T. y TAKAO, M., 1994: “Perception” en The brain, our universe within (serie para TV), NHK/NHK CREATIVE, inc., Tokio

- SCHAEFFER, P., 1988: Tratado de los objetos musicales, Alianza, Madrid.

- VARELA, F.J. (edic.), 1998: El sueño, los sueños y la muerte, J.J. de Olañeta, Palma de Mallorca.

- WATTS, A., 1995: Qué es la realidad, Kairós, Barcelona.

- $\quad$ WILBER, K., 1994; Psicología integral, Kairós, Barcelona. 